\section{The frequency of Human Papillomavirus Genotypes in Invasive Cervical Cancer of Romanian Patients}

\section{DOI: $10.1515 / \mathrm{rrlm}-2017-0008$}

Cervical cancer, statistically, occupies the fourth place worldwide among cancers in women in term of incidence and mortality; most of the cases are discovered in developing countries (1). Its etiology is related to Human papillomavirus (HPV) infection, a necessary, but insufficient cause of most of the cervical cancers, but also for a high proportion of vulva, vagina or anal cancers, and some of the oropharyngeal cancers (2). Currently, more than 200 HPV subtypes have been isolated, and more than 40 different HPV types are found in the genital tract or perianal region (3). The condition of HPV infection to cause a cervical cancer is to become persistent and to escape from the immune defence of the host. Data about the frequency of HPV infection in Romanian population are scanty. Only few studies have been done (4-7), and they focused mainly on cytological abnormalities and pre-invasive cervical lesions, but not in invasive cervical cancer. Thus, the goal of our research was to obtain preliminary information about the frequency of HPV infection and the HPV genotypes in a Romanian population with invasive cervical cancer.

The samples included 80 histologically confirmed invasive cervical cancer fresh specimens of previously non-irradiated Romanian women, collected between September 2015 and October 2016 in a tertiary university hospital. The Ethics Committee of the hospital approved the study protocol. The patients were staged according to the International Federation of Gynecology and
Obstetrics (FIGO) staging. All specimens were collected with PreservCyt $t^{\mathrm{T}}$ and stored at $+4^{\circ} \mathrm{C}$ until analysed. Detection consisted in genomic DNA extraction from cervical epithelial cells, PCR amplification of target DNA (37 HPV genotypes including 13 high risk genotypes: 16,18 , $31,33,35,39,45,51,52,56,58,59$, and 68), hybridization of PCR products with oligonucleotide probes on solid strips and colorimetric identification of hybridization productsaccording to manufacturer recommendations (LINEAR ARRAY HPV Genotyping Test, Roche). A chi square test and a logistic regression were performed to try to find a correlation between HPV type, stage of disease, or histological type. Statistical analyses for $P$ values were performed using $2 \times 2$ contingency tables, with two-tailed $P$ values calculated using the Fisher exact test. All $p<0.05$ were considered statistically significant. The patients' mean age at the time of diagnosis was 49 years (between 28 and 68 years old). The majority of histological diagnoses were squamous cell carcinoma ( 75 out of 80 patients, $93.75 \%$ ), with 4 adenocarcinomas $(5 \%)$ and one neuroendocrine carcinoma. Forty-one patients $(51.25 \%)$ were stage I (Ia1-Ib2), 24 (30\%) stage II, 14 (17.5\%) stage III, and one $(1.25 \%)$ was staged IVa. Of the 80 samples analyzed, 10 cases $(12.5 \%)$ were negative for HPV-DNA. Most of HPV-DNA positive cervical cancer patients harboured single infections $(87.14 \%) ; 7$ patients had two HPV types $(10 \%)$; and in 2 patients $(2.86 \%)$, three different HPV types were identified. HPV16 was the most frequent type, with $72.5 \%$ relative contribution as single (58 patients) or multiple infection (2 cases of double infection and 2 patients harbouring three different HPV types). Other two HPV double infections harboured HPV31 associated with HPV39 and HPV73. Interestingly, HPV18 
was identified in only one biopsy (1.3\%). More high risk HPV types were found in 2 cases (HPV $31,33,39,45,53,58,62,68)$. One low risk HPV (type 6) was identified. No statistical correlation was found between the HPV genotype, patients' age, histology of the tumour, or the stage of the disease. Lack of available information regarding the prevalence of HPV infections in Romania makes the acceptance of the HPV vaccination campaign by the population more difficult. In a country with a high incidence of cervical cancer, with a less organized national screening programme, more data regarding the frequency of HPV infection could sustain the need to continue the HPV vaccination, against its misinformed opponents, influenced also by religious or cultural reasons. The HPV frequency in cervical cancer patients differs in different world regions. In studies performed in Portugal and Croatia $(8,9)$, HPV prevalence was $97.9 \%$, and $92.59 \%$ respectively. Regarding Asian populations, HPV positivity ranged between $64.8 \%$ $-90.1 \%$ in different regions of Thailand (10), to $97.6 \%$ in squamous cell carcinoma cases in China (11). In a meta-analysis performed on studies from Malaysia, Vietnam, Singapore, South Korea, and the Philippines, HPV frequency ranged from $84.5 \%$ to $97 \%$ (12). In sub-Saharan Africa, HPV-positivity was observed in $90.4 \%$ of cases (13) The prevalence of HPV 16 is higher in Europe, South America, and China compared to other countries; by contrast, HPV 18, 45, and 52 is more frequent in South East Asia compared to the rest of the world. Multiple infections were observed in $1.7-52.2 \%$ of the cases $(8,13,14)$. In a retrospective study (15), de Sanjose et al. analyzed paraffin-embedded samples of confirmed invasive cervical carcinoma from 38 countries worldwide. In Europe, the most frequent type identified was HPV $16(66 \%)$, followed by HPV 18 positive cases (7\%), and HPV 33 positive cases $(6 \%)$. This ranking of genotypes was maintained when samples from North America were analyzed. In samples analyzed from Asia and Central and South America the third most common type was HPV 45. In African populations, a more heterogeneous genotyping was found: after HPV 16 and HPV 18, the frequency of HPV types 45, 52, 58, 31, and 33 was close in percentages to the HPV heterogeneity found in the Romanian population.

Our results showed a high prevalence of HPV 16 single infection (72.5\%), and multiple infections in $12.85 \%$ of women. HPV 18 was detected in only one patient, despite the presence of 4 adenocarcinomas and one neuroendocrine carcinoma histology. This high variety of HPV types in a Romanian population is quite specific, compared to other recently published data. In this regard, HPV type 16 and 18 accounted for $92.1 \%$ of invasive cervical cancer patients sampled in Tunisia (14). Single infection was found in $48.8 \%$ of the patients. The other oncogenic HPV single infections were associated with HPV 35 (4.6\%), 45 (4.6\%), 58 (2.3\%), and $59(2.3 \%)$. Multiple infections with mixing of 2 to 4 genotypes predominately featured HPV16 and/ or 18 with HPV 35 and 45 (96.6\%). No statistical correlation was found between the distribution of HPV types and patients' age. A pooled analysis study performed in Italy included 574 invasive cervical cancers (16). HPV 16 genotype was the most frequent type, in single $(69.3 \%)$ and in co-infections with other genotypes $(58.3 \%)$, in all squamous cell carcinomas and adenocarcinomas. However, HPV 18 type was the second most common genotype linked with invasive cervical carcinoma (23.3\%); HPV 18 positive infections (were in low proportion in our group $(2.13 \%)$. This trend is also confirmed with other HPV HR types: 45, 31, 58, 33.

In a study from Pakistan (17), among the HPV positive cases with cervical cancer, in 95.9\% a single HPV type was isolated: HPV 
16 was the most frequent, followed by HPV 18 and 45. In women from Israel with cervical cancer (18), high risk HPV types were detected in $93 \%$. The most common HPV type was 16 (57.4\%), followed by HPV 45 (9.6\%), and HPV $18(7.8 \%)$. Multiple HPV types were isolated in $0.9 \%$ of women. In a study from Northern Ireland (19), HPV was isolated in $92.2 \%$ of squamous cell carcinomas, and $64.3 \%$ of adenocarcinoma patients, respectively. Most squamous cell carcinomas histologies $(81.3 \%)$ had only one HPV type detected. In a recently published meta-analysis (20), the prevalence of HPV16 and HPV18 genotypes declined significantly with age among women with cervical cancer from $74.8 \%$ in the $30-39$ years age group to $56.8 \%$ in the over 70 years old group. A very interesting result derived from this study: $12.5 \%$ of the population was diagnosed with invasive cervical carcinoma with a negative HPV genotyping result. Some researchers explained in their studies that they cannot exclude technical artefacts that could occur in HPV-DNA genotyping, for example poor DNA quality and PCR inhibition (15), yet a number of cases of cervical squamous cell carcinoma and adenocarcinomas might appear independent of HPV infections.

\section{Acknowledgement}

This study was supported by internal research grant 12/23.12.2014 offered by the University of Medicine and Pharmacy of Tîrgu Mureș, Romania. The authors have no conflicts of interest to disclose.

Mihai Emil Căpîlna and Romeo Micu contributed equally to this research.

\section{Mihai Emil Căpîlna ${ }^{1}$, Septimiu Voidăzan 2,*, Mihaela Alexandra Budianu', János Bécsi ${ }^{1}$, Romeo Micu ${ }^{3}$, Ștefan Barbu ${ }^{4}$}

1. First Obstetrics and Gynecology Clinique, University of Medicine and Pharmacy, Târgu Mureș, Romania;

2. Department of Epidemiology, University of Medicine and Pharmacy, Târgu Mureș, Romania;

3. First Obstetrics and Gynaecology Clinic, University of Medicine and Pharmacy "Iuliu Hațieganu”, Cluj-Napoca, Romania;

4. Central Medical Laboratory, Emergency Hospital Tirgu Mures, Romania.

* Corresponding author: Septimiu Voidăzan, e-mail: septimiu.voidazan@rrml.ro

Received: $9^{\text {th }}$ January 2017; Accepted: $31^{\text {th }}$ January 2017; Published: 03 ${ }^{\text {th }}$ April 2017.

\section{References}

1. Ferlay J., Soerjomataram I., Ervik M., Dikshit R., Eser S., Mathers C., et al. International Agency for Research on Cancer; Lyon, France: 2013. GLOBOCAN 2012 v1.0, Cancer incidence and mortality worldwide: IARC Cancer Base No. 11. Available online: http://globocan.iarc.fr.

2. Wentzensen N, Arbyn M, Berkhof J, Bower M, Canfell K, Einstein M, et al. Eurogin 2016 Roadmap: How HPV knowledge is changing screening practice. Int J Cancer. 2016 Dec 22. [Epub ahead of print] DOI: $10.1002 /$ ijc.30579

3. Castellsagué X, Díaz M, de Sanjosé S, Mu-oz N, Herrero $\mathrm{R}$, Franceschi S, et al. Worldwide human papillomavirus etiology of cervical adenocarcinoma and its cofactors: Implications for screening and prevention. J. Natl. Cancer Inst. 2006;98:303-315. DOI: 10.1093/jnci/djj067

4. Ursu RG., Onofriescu M, Nemescu D, Iancu LS. HPV prevalence and type distribution in women with or without cervical lesions in the Northeast region of Romania. Virol J. 2011;8:558. DOI: 10.1186/1743-422X-8-558

5. Melinte-Popescu A., Costăchescu G. Human papilloma virus infection and cervical dysplasia. Rev Med Chir Soc Med Nat Iasi. 2012;116(3):853-857.

6. Feticu L., Bocşan I.S., Bondor C.I., Boboş C. Geographical distribution of human papillomavirus and their correlation with cytologic abnormalities in cervical lesions and with the age in a female population from Transylvania. Rev Med Chir Soc Med Nat Iasi. 2012;116(1):291-298. 
7. Voidăzan S, Morariu SH, Căpîlna M, Mărginean C, Dobreanu M. The prevalence and distribution of high risk Human Papillomavirus genotypes in patients with dysplastic lesions: a population study. Acta Medica Marisiensis 2015;61(4):314-319.

8. Pista A, de Oliveira CF, Lopes C, Cunha MJ; CLEOPATRE Portugal Study Group. Human papillomavirus type distribution in cervical intraepithelial neoplasia grade $2 / 3$ and cervical cancer in Portugal: a CLEOPATRE II Study. Int J Gynecol Cancer. 2013 Mar;23(3):500-6. DOI: 10.1097/ IGC.0b013e318280f26e

9. Hadzisejdić I, Simat M, Bosak A, Krasević M, Grahovac B. Prevalence of human pa-pillomavirus genotypes in cervical cancer and precursor lesions. Coll Antropol. 2006 Dec;30(4):879-83.

10. Kietpeerakool C, Kleebkaow P, Srisomboon J. Human Papillomavirus Genotype Dis-tribution among Thai Women with High-Grade Cervical Intraepithelial Lesions and Invasive Cervical Cancer: a Literature Review. Asian Pac J Cancer Prev. 2015;16(13):5153-8. DOI: 10.7314/APJCP.2015.16.13.5153

11. Chen W, Zhang X, Molijn A, Jenkins D, Shi JF, Quint $\mathrm{W}$, et al. Human papillomavirus type-distribution in cervical cancer in China: the importance of HPV 16 and 18. Can-cer Causes Control. 2009 Nov;20(9):1705-13. DOI: $10.1007 / \mathrm{s} 10552-009-9422-\mathrm{z}$

12. Quek SC, Lim BK, Domingo E, Soon R, Park JS, Vu $\mathrm{TN}$, et al. Human papillomavirus type distribution in invasive cervical cancer and high-grade cervical intraepithelial neo-plasia across 5 countries in Asia. Int J Gynecol Cancer. 2013 Jan;23(1):148-56. DOI: 10.1097/IGC.0b013e31827670fd

13. Denny L, Adewole I, Anorlu R, Dreyer G, Moodley M, Smith T, et al. Human papil-lomavirus prevalence and type distribution in invasive cervical cancer in sub-Saharan Africa. Int J Cancer. 2014 Mar 15;134(6):138998. DOI: $10.1002 /$ ijc. 28425

14. Ennaifer E, Salhi F, Laassili T, Fehri E, Ben Alaya N, Guizani I, et al. Type-specific Human Papillomavirus distribution in invasive squamous cervical carcinomas in Tunisia and vaccine impact. Asian Pac J Cancer Prev. 2015;16(15):6769-72. DOI: 10.7314/ APJCP.2015.16.15.6769
15. de Sanjose S, Quint WG, Alemany L, Geraets DT, Klaustermeier JE, Lloveras B, et al. Human papillomavirus genotype attribution in invasive cervical cancer: a retrospective cross-sectional worldwide study. Lancet Oncol. 2010;11(11):1048-56. DOI: 10.1016/S14702045(10)70230-8

16. Giorgi Rossi P, Sideri M, Carozzi FM, Amina Vocaturo, Franco Maria Buonaguro, Maria Lina Tornesello, et al. HPV type distribution in invasive cervical cancers in Italy: pooled analysis of three large studies. Infectious Agents and Cancer. 2012;7:26. DOI: 10.1186/17509378-7-26

17. Loya A, Serrano B, Rasheed F, Tous S, Hassan M, Clavero O, et al. Human Papillo-mavirus genotype distribution in invasive cervical cancer in Pakistan. Cancers (Basel). 2016 Jul 30;8(8). pii: E72. DOI: $10.3390 /$ cancers 8080072

18. Siegler E, Sharir K, Lavie O, Saked-Misan P, Machulki L, Auslender R, et al. The pre-valence of HPV types in women with CIN 2-3 or cervical cancer in Haifa District, Is-rael. Minerva Ginecol. 2016 Sep 16. [Epub ahead of print]

19. Anderson LA, O'Rorke MA, Wilson R, Jamison J, Gavin AT Northern Ireland HPV Working Group. HPV prevalence and type-distribution in cervical cancer and prema-lignant lesions of the cervix: A population-based study from Northern Ireland. J Med Virol. 2016 Jul;88(7):1262-70. DOI: 10.1002/jmv.24447

20. Hammer A, Rositch A, Qeadan F, Gravitt PE, Blaakaer J. Age-specific prevalence of HPV16/18 genotypes in cervical cancer: A systematic review and meta-analysis. Int J Cancer. 2016 Jun 15;138(12):2795-803. DOI: 10.1002/ijc. 29959 